\title{
Estimation of Carrier Frequency Offset for Multicarrier CDMA Uplink
}

\author{
Yi Ma, Member, IEEE, and Rahim Tafazolli
}

\begin{abstract}
This paper presents a training-based estimation method of carrier frequency offset (CFO) for multicarrier codedivision multiple-access (MC-CDMA) uplink. The basic assumption is the same as in Morellis' work (OFDMA), i.e., active users in the network are classified into two categories: reference (synchronized) users (RUs) and new (asynchronized) users (NUs). But, we consider the system that RUs use CDMA to share the wireless medium. NUs employ time-division multiple-access (TDMA) for the training purpose. Our major idea is to perform the CFO estimation in the desired signal subspace that is orthogonal to the RU subspace. The proposed approach is investigated both in the subband and interleaved MC-CDMA systems. It is shown that implementation of the CFO estimation together with the training-pattern design could be relatively simple when users do not experience the considerable frequency-selective fade. In the highly frequency-selective fading scenario, an adaptive training-scheme is proposed to make the CFO estimator robust. We also consider NUs operating in the frequency-division multiple-access (FDMA) training mode. Due to the presence of inter-NU-interference, the estimation performance may be not as good as in the TDMA training mode. The performance degradation may be severe when RUs operate in the interleaved MC-CDMA. In this case, we propose an inter-NU-interference self-canceling training-pattern and use simulations to demonstrate its impact on the estimation performance.
\end{abstract}

Index Terms-Carrier frequency offset (CFO), multicarrier code-division multiple-access (MC-CDMA), singular-value decomposition (SVD), symbol timing offset, uplink.

\section{INTRODUCTION}

$\mathbf{E}$ STIMATION of carrier frequency offset (CFO) for multicarrier (MC) systems has been intensively investigated in recent years. Most of CFO estimation approaches reported so far were proposed for the downlink (single-user equivalent scenario) (e.g., [1] and [2]). Applying MC technique in wireless networking or cellular radio calls for the CFO estimation for the uplink (multi-user scenario), where each user has its own $\mathrm{CFO}$. The pioneer research on the $\mathrm{CFO}$ estimation for the $\mathrm{MC}$ uplink might be conducted by van de Beek et al. (see [3]). Their work focused on orthogonal frequency-division multiple-access (OFDMA) system. The major idea was to combine the cyclic prefix (CP)-based blind estimation approach (see [4]) with the

Manuscript received April 10, 2006; revised July 13, 2006. This work was supported by the European Commission under IST 4More Project IST-2002507039. The associate editor coordinating the review of this manuscript and approving it for publication was Dr. Erchin Serpedin.

The authors are with the Center for Communication Systems Research, University of Surrey, Surrey GU2 7XH, U.K. (e-mail: y.ma@surrey.ac.uk; r.tafazolli@ surrey.ac.uk).

The associate editor coordinating the review of this paper and approving it for publication was Dr. Erchin Serpedin.

Digital Object Identifier 10.1109/TSP.2006.890883 bandpass-filterbank-based user separation. Recently, a subspace-based blind approach was proposed for the interleaved OFDMA, which exploits the time-domain cyclo-stationarity of the signal structure (see [5]). Besides the blind approaches, the training-based approaches are practically used in wireless systems. The recent result on this aspect was reported by Morelli in [6] that also focused on the OFDMA uplink. It was assumed that the active users in the network can be classified into reference (synchronized) users (RUs) and new (asynchronized) users (NUs). NUs do not share the common OFDM block at the training stage to avoid the inter-NU-interference. Since RUs do not interfere NUs in the frequency domain, the CFO estimation algorithm for the single-user scenario (as in [1]) can be directly applied in the NU subspace. To protect the assumption, the CFO, as well as the timing offset, must be compensated at the transmitter.

The question is, can we apply the existing approaches (uplink) into the MC system that users use the linear precoding (or called code spreading) technique? A good summary of the MC system with the linear precoder (LP), so called generalized MC system, can be found in [7]. Early work on the MC-LP system was documented in [8] and [9] that proposed several typical frameworks, namely MC-SS-MA, MC-DS-CDMA as well as MC-CDMA. Actually, MC-SS-MA is an upgraded version of the OFDMA, where users do not share the common subcarriers. Therefore, the CFO estimation approaches for the OFDMA uplink can be directly (or with minor modification) applied in the MC-SS-MA system. The others either spread the user data along the time-direction (MC-DS-CDMA) or along the frequency-direction (MC-CDMA). Their common feature is that users share the common subcarriers. In this case, the introduced blind or training-based approaches relying on the OFDMA structure are not applicable.

In this paper, we propose a training-based CFO estimation approach for the MC-CDMA uplink, where RUs' data are spread over all subcarriers. In the inter-NU-interference free case, the training sequences sent by NUs may occupy all subcarriers. At the receiving end, the training sequences are superimposed onto the RUs' data. It is assumed that the uplink receiver knows the effective RU signatures. ${ }^{1}$ Thanks to the precoding redundancy, we can perform the singular-value decomposition (SVD) on the RU signature matrices to find the desired subspace which is orthogonal to the RU subspace. Then, the CFO estimation can be carried out in the desired subspace. The basic idea here can be regarded as the generalization of Morelli's approach. However, the CFO identifiability needs to be carefully investigated in the MC-CDMA system. We find that the implemen-

\footnotetext{
${ }^{1}$ This is a reasonable assumption, since the effective RU signatures are needed for the signal detection.
} 


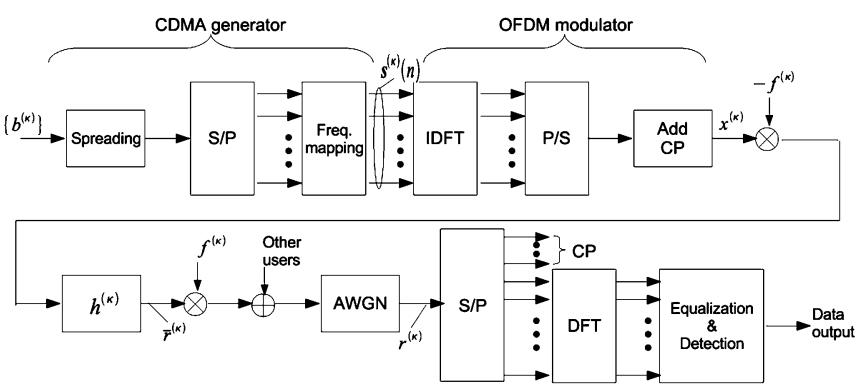

Fig. 1. Block diagram of the MC-CDMA uplink.

tation of the CFO estimation as well as the training-pattern design could be relatively simple in the subband MC-CDMA. In the highly frequency-selective scenario, such as the interleaved MC-CDMA, we propose an adaptive training scheme to make the estimator robust. The mean-square error (MSE) bound as well as Modified Cramér-Rao bound (MCRB) is given to examine the estimation performance. The final BER is examined via simulations with the $\mathrm{CFO}$ compensation at the transmitting end. We also investigate the CFO estimation in the inter-NU-interference context, where NUs use FDMA to share the common OFDM block. It is observed that the estimation performance may not be as good as in the inter-NU-interference free case. To cope with the inter-NU-interference, we borrow the idea from [10] and propose an interference self-canceling training pattern. The inter-NU-interference canceling capability of the proposed training pattern is carefully investigated in the Gaussian channel with the presence of timing offset. Computer simulations are used to show the improved estimation performance.

The rest of this paper is organized as follows. Section II gives the system model of MC-CDMA uplink. The CFO estimation in the inter-NU-interference free case is presented in Section III. Section IV considers the CFO estimation in the presence of inter-NU-interference. Simulation results are provided in Section V. Section VI draws the conclusion.

\section{MC-CDMA UPLINK SYSTEM}

\section{A. MC-CDMA System}

We consider a CP-OFDM system with $M$ subcarriers and accommodating a maximum of $\mathcal{K}$ active users. As depicted in Fig. 1, each user transmits the symbols $\left\{s^{(\kappa)}\right\}$ $(\kappa)$ for user index), the output of the CDMA generator, over all subcarriers. ${ }^{2}$ Prior to transmission, the symbols $\left\{s^{(\kappa)}\right\}$ are first grouped into $M \times 1$ blocks $\boldsymbol{s}^{(\kappa)}(n)=$ $\left[s^{(\kappa)}(n M), s^{(\kappa)}(n M+1), \ldots, s^{(\kappa)}(n M+M-1)\right]^{\mathrm{T}}\left({ }^{\mathrm{T}}\right.$ for transpose). These blocks are fed into the CP-OFDM modulator to produce

$$
\begin{aligned}
& x^{(\kappa)}(n J+i)=\frac{1}{\sqrt{M}} \sum_{m=0}^{M-1} s^{(\kappa)}(n M+m) \\
& \times e^{\frac{j 2 \pi m(i+M-P)}{M}}, \quad 0 \leqslant i \leqslant J-1 \\
& J=M+P
\end{aligned}
$$

\footnotetext{
${ }^{2} \mathrm{An}$ OFDM system usually employs some virtual carriers (see [2]). Here, we ignore this technical point for convenience.
}

where $n$ stands for block index, $m$ for subcarrier index, $i$ for sample index, and $P$ for the number of samples in CP. Then, $x^{(\kappa)}$ goes through the communication channel $h^{(\kappa)}$ with the upper bound of channel order $L_{h}(\leqslant P)$, and experiences the frequency offset $f^{(\kappa)}$ as well as the propagation delay $\tau^{(\kappa)}$. The received discrete-time equivalent signal is (see [6])

$$
\begin{aligned}
r(n J+i)=\sum_{\kappa=1}^{\mathcal{K}} e^{\frac{j 2 \pi \nu(\kappa)(n J+i)}{M}} \bar{r}^{(\kappa)} & \\
& \times\left(n J+i-\mu^{(\kappa)}\right)+v(n J+i)
\end{aligned}
$$

where $\bar{r}^{(\kappa)}$ is the signal from the $\kappa$ th user

$$
\bar{r}^{(\kappa)}(n J+i)=\sum_{\imath=-\infty}^{\infty} x^{(\kappa)}(\imath) h^{(\kappa)}(n J+i-\imath)
$$

$v$ the zero-mean Gaussian noise, $\nu^{(\kappa)}$ the normalized version of $f^{(\kappa)}$, i.e., $\nu^{(\kappa)}=f^{(\kappa)} M T_{s}$ (Ts for sampling period), and $\mu^{(\kappa)}=\left\lfloor\left(\tau^{(\kappa)}\right) /\left(T_{s}\right)\right\rfloor$ the integer part of timing offset $(\lfloor\cdot\rfloor$ the integer floor). The fraction part of $\left(\tau^{(\kappa)}\right) /\left(T_{s}\right)$ is incorporated into $h^{(\kappa)}$ (see [6] for reasons).

We assume, but only here, that all active users are well synchronized, i.e., $\nu^{(\kappa)}=0, \mu^{(\kappa)}=0,(\kappa=1,2, \ldots, \mathcal{K}-1)$. The receiver discards the CP part and performs the discrete Fourier-transform (DFT) on $r(n J+i), i=P, P+1, \ldots, P+$ $M-1$, to obtain the frequency-domain version of the received signal

$$
\begin{array}{r}
\tilde{r}(n M+m)=\sum_{\kappa=1}^{\mathcal{K}} \tilde{h}^{(\kappa)}(m) s^{(\kappa)}(n M+m)+\tilde{v}(n M+m), \\
0 \leqslant m \leqslant M-1
\end{array}
$$

where $\tilde{h}^{(\kappa)}(m)=\sum_{l=0}^{L_{h}} h^{(\kappa)}(l) \exp (-j 2 \pi l m / M)$ and $\tilde{v}$ the frequency-domain version of the noise. We can see that the received signal is the superposition of all active users. Symbol detection on (4) needs the channel state information as well as a proper design of $s^{(\kappa)}$.

\section{B. CDMA Generator and Subcarrier Mapping}

CDMA generator together with subcarrier (frequency) mapping strategy relates the information symbols $\left\{b^{(\kappa)}\right\}$ to the blocks $\boldsymbol{s}^{(\kappa)}$. We first consider that each active user only transmits one information symbol $b^{(\kappa)}$ over $\bar{M}$ out of $M$ subcarriers $(\bar{M} \geqslant \mathcal{K}, Q=M / \bar{M}$ is an integer). The spreading code $\boldsymbol{c}^{(\kappa)}=\left[c^{(\kappa)}(0), c^{(\kappa)}(1), \ldots, c^{(\kappa)}(\bar{M}-1)\right]^{\mathrm{T}}$ is drawn from the $\bar{M} \times \bar{M}$ Walsh-Hadamard matrix [11]. Users do not use identical spreading code. There are two typical subcarrier mapping methods. One is "subband" mapping approach that allocates the result $c^{(\kappa)} b^{(\kappa)}$ on the subcarriers $\{q \bar{M}, q \bar{M}+1, \ldots, q \bar{M}+\bar{M}-1\},(q=0,1, \ldots, Q-1)$, the other is "interleaved" mapping approach that allocates $\boldsymbol{c}^{(\kappa)} b^{(\kappa)}$ on the subcarriers $\{q, q+Q, \ldots, q+(\bar{M}-1) Q\}$. The later one turns out to be the optimal mapping approach [12].

We can see the amount of subcarriers are divided into $Q$ subsets. Each user can transmit $Q$ symbols per block over these 
subsets. The received signal in the $q$ th subset (for both subband and interleaved approaches) has the unified formula

$\tilde{\boldsymbol{r}}_{q}(n)=\sum_{\kappa=1}^{\mathcal{K}} \mathbf{D}\left(\tilde{\boldsymbol{h}}_{q}^{(\kappa)}\right) \boldsymbol{c}^{(\kappa)} b_{q}^{(\kappa)}(n)+\tilde{\boldsymbol{v}}_{q}(n)$,

$$
q=0,1, \ldots, Q-1
$$

where the subscript ${ }_{q}$ denotes the subset index, $\tilde{\boldsymbol{h}}_{q}^{(\kappa)}$ collects the channel information for the $\kappa$ th user in the $q$ th subset, i.e., $\tilde{\boldsymbol{h}}_{q}^{(\kappa)}=\left[\tilde{h}^{(\kappa)}(q \bar{M}), \tilde{h}^{(\kappa)}(q \bar{M}+1), \ldots, \tilde{h}^{(\kappa)}(q \bar{M}+\bar{M}-1)\right]^{\mathrm{T}}$ for the subband approach, or $\tilde{\boldsymbol{h}}_{q}^{(\kappa)}=\left[\tilde{h}^{(\kappa)}(q), \tilde{h}^{(\kappa)}(q+\right.$ $\left.Q), \ldots, \tilde{h}^{(\kappa)}(q+(\bar{M}-1) Q)\right]^{\mathrm{T}}$ for the interleaved approach, $\tilde{\boldsymbol{v}}_{q}$ the corresponding noise, $\mathbf{D}(\boldsymbol{a})$ the diagonal matrix with $\boldsymbol{a}$ in its diagonal. Let $\tilde{\boldsymbol{c}}_{q}^{(\kappa)}=\mathbf{D}\left(\tilde{\boldsymbol{h}}_{q}^{(\kappa)}\right) \boldsymbol{c}^{(\kappa)}$ denote the effective user signature, and define $\tilde{\boldsymbol{C}}_{q}=\left[\tilde{\boldsymbol{c}}_{q}^{(1)}, \tilde{\boldsymbol{c}}_{q}^{(2)}, \ldots, \tilde{\boldsymbol{c}}_{q}^{(\mathcal{K})}\right]$ and $\boldsymbol{b}_{q}(n)=\left[b_{q}^{(1)}(n), b_{q}^{(2)}(n), \ldots, b_{q}^{(\mathcal{K})}(n)\right]^{\mathrm{T}}$. We can rewrite (5) into the following matrix form:

$$
\tilde{\boldsymbol{r}}_{q}(n)=\tilde{\boldsymbol{C}}_{q} \boldsymbol{b}_{q}(n)+\tilde{\boldsymbol{v}}_{q}(n), \quad q=0,1, \ldots, Q-1 .
$$

With the knowledge of $\tilde{\boldsymbol{C}}_{q}$, we can carry out the zero-forcing (ZF) equalization

$$
\begin{aligned}
\boldsymbol{z}_{q}(n) & =\tilde{\boldsymbol{C}}_{q}^{\dagger} \tilde{\boldsymbol{r}}_{q}(n) \\
& =\boldsymbol{b}_{q}+\tilde{\boldsymbol{C}}_{q}^{\dagger} \tilde{\boldsymbol{v}}_{q}(n)
\end{aligned}
$$

where ${ }^{\dagger}$ denotes pseudo inverse. (8) holds only when $\tilde{C}_{q}$ has the full column rank, i.e., $\bar{M} \geqslant \mathcal{K}$ is necessary. Next, we use (6) to formulate the received RUs signal, and investigate the CFO estimation for NUs.

\section{CFO ESTIMATION Without INTER-NU-INTERFERENCE}

\section{A. Training Scheme}

We consider $\mathcal{K}_{r}(<\mathcal{K})$ RUs already in the network. An NU with the user index $k$ just enters the network and sends $N$ identical training blocks for the CFO estimation. In this context, the received time-domain signal is the superposition of the RUs and the NU signal

$$
\begin{aligned}
r(n J+ & i)=\left(\sum_{\kappa=1}^{\mathcal{K}_{r}} \bar{r}^{(\kappa)}(n J+i)\right) \\
& +\underbrace{e^{\frac{j 2 \pi \nu^{(k)}(n J+i)}{M}} \bar{r}^{(k)}\left(n J+i-\mu^{(k)}\right)}_{=y^{(k)}\left(n J+i-\mu^{(k)}\right)}+v(n J+i) .
\end{aligned}
$$

Let $y^{(k)}\left(n J+i-\mu^{(k)}\right)$ be the input to the OFDM demodulator, the output is [13]

$$
\begin{aligned}
\tilde{y}^{(k)}(n M+m)= & e^{\frac{j 2 \pi \nu^{(k)}(n J+P)}{M}} \xi^{(k)}(m), 0 \leqslant m \leqslant M-1 \\
\xi^{(k)}(m)= & \underbrace{e^{\frac{j 2 \pi m \mu^{(k)}}{M}} \tilde{h}^{(k)}(m) t^{(k)}(m) d(0)}_{=\alpha^{(k)}(m)} \\
& +\underbrace{\sum_{\ell=0, \ell \neq m}^{M-1} e^{\frac{j 2 \pi \ell \mu}{M}} \tilde{h}^{(k)}(\ell) t^{(k)}(\ell) d(\ell-m)}_{=\beta^{(k)}(m)}
\end{aligned}
$$

where

$$
\begin{aligned}
d(\ell-m)= & \frac{\sin \left(\pi\left(\ell-m+\nu^{(k)}\right)\right)}{M} \sin \left(\pi\left(\ell-m+\nu^{(k)}\right) / M\right) \\
& \cdot \exp \left(j \pi(1-1 / M)\left(\ell-m+\nu^{(k)}\right)\right)
\end{aligned}
$$

$t^{(k)}$ denotes the training symbols, $\alpha^{(k)}$ the signal term, $\beta^{(k)}$ the inter-carrier interference (ICI). Then, the output of OFDM demodulator with the input $r(n J+i)$ is the superposition of (4) and (10)

$$
\begin{aligned}
& \tilde{r}(n M+m)=\left(\sum_{\kappa=1}^{\mathcal{K}_{r}} \tilde{h}^{(\kappa)}(m) s^{(\kappa)}(n M+m)\right) \\
&+e^{\frac{j 2 \pi \nu(k)(n J+P)}{M}} \xi^{(k)}(m)+\tilde{v}(n M+m), \\
& 0 \leqslant m \leqslant M-1 .
\end{aligned}
$$

The received signal within the $q$ th subset is expressible as

$$
\begin{array}{r}
\tilde{\boldsymbol{r}}_{q}(n)=\tilde{\boldsymbol{C}}_{q} \boldsymbol{b}_{q}(n)+e^{\frac{j 2 \pi \nu^{(k)}(n J+P)}{M}} \boldsymbol{\xi}_{q}^{(k)}+\tilde{\boldsymbol{v}}_{q}(n), \\
q=0,1, \ldots, Q-1 .
\end{array}
$$

Here, the size of $\tilde{C}_{q}$ is $\bar{M} \times K_{r}$, and $\xi_{q}^{(k)}=$ $\left[\xi^{(\kappa)}(q \bar{M}), \xi^{(\kappa)}(q \bar{M}+1), \ldots, \xi^{(\kappa)}(q \bar{M}+\bar{M}-1)\right]^{\mathrm{T}}$ for the subband MC-CDMA, or $\boldsymbol{\xi}_{q}^{(k)}=\left[\xi^{(\kappa)}(q), \xi^{(\kappa)}(q+\right.$ $\left.Q), \ldots, \xi^{(\kappa)}(q+(\bar{M}-1) Q)\right]^{\mathrm{T}}$ for the interleaved MC-CDMA. Our objective is to estimate $\nu^{(k)}$ with the knowledge of $\tilde{\boldsymbol{C}}_{q}$ available at the receiver.

\section{B. CFO Estimation}

We start from the SVD of $\tilde{\boldsymbol{C}}_{q}[14]$

$$
\left[\tilde{\boldsymbol{C}}_{q}, \mathbf{0}\right]_{\bar{M} \times \bar{M}}=\boldsymbol{U}_{q}\left[\begin{array}{ll}
\boldsymbol{\Lambda}_{q} & \\
& \mathbf{0}
\end{array}\right] \boldsymbol{V}_{q}^{\mathcal{H}}
$$

where $\boldsymbol{U}_{q}, \boldsymbol{V}_{q}$ is the $\bar{M} \times \bar{M}$ unitary matrix, the central matrix on the right hand is diagonal, and $\Lambda_{q}$ the $\mathcal{L}_{\Lambda} \times \mathcal{L}_{\Lambda}$ diagonal matrix $\left(\mathcal{L}_{\Lambda} \leqslant \mathcal{K}_{r}\right)$. Therefore, we have a matrix $\overline{\boldsymbol{U}}_{q}$ formed by the last $\left(\bar{M}-\mathcal{K}_{r}\right)$ columns of $\boldsymbol{U}_{q}$, which fulfills

$$
\overline{\boldsymbol{U}}_{q}^{\mathcal{H}} \tilde{\boldsymbol{C}}_{q}=\mathbf{0} .
$$

Multiplying $\overline{\boldsymbol{U}}_{q}^{\mathcal{H}}$ with $\tilde{\boldsymbol{r}}_{q}(n)$ filters out the reference users

$$
\begin{aligned}
& \overline{\tilde{\boldsymbol{r}}}_{q}(n)=\overline{\boldsymbol{U}}_{q}^{\mathcal{H}} \tilde{\boldsymbol{r}}_{q}(n) \\
& =e^{\frac{j 2 \pi \nu(k)}{M}(n J+P)} \underbrace{\overline{\boldsymbol{U}}_{q}^{\mathcal{H}} \boldsymbol{\xi}_{q}^{(k)}}_{=\overline{\boldsymbol{\xi}}_{q}^{(k)}}+\underbrace{\overline{\boldsymbol{U}}_{q}^{\mathcal{H}} \tilde{\boldsymbol{v}}_{q}(n)}_{=\overline{\boldsymbol{v}}_{q}(n)} .
\end{aligned}
$$

Define vectors $\overline{\boldsymbol{r}}(n)=\left[\overline{\tilde{\boldsymbol{r}}}_{0}^{\mathrm{T}}(n), \overline{\tilde{\boldsymbol{r}}}_{1}^{\mathrm{T}}(n), \ldots, \overline{\tilde{\boldsymbol{r}}}_{Q-1}^{\mathrm{T}}(n)\right]^{\mathrm{T}}$, $\overline{\boldsymbol{\xi}}^{(k)}=\left[\left(\overline{\boldsymbol{\xi}}_{0}^{(k)}\right)^{\mathrm{T}},\left(\overline{\boldsymbol{\xi}}_{1}^{(k)}\right)^{\mathrm{T}}, \ldots,\left(\bar{\xi}_{Q-1}^{(k)}\right)^{\mathrm{T}}\right]^{\mathrm{T}}, \quad \overline{\boldsymbol{v}}(n)=$ $\left[\overline{\tilde{\boldsymbol{v}}}_{0}^{\mathrm{T}}(n), \overline{\tilde{\boldsymbol{v}}}_{1}^{\mathrm{T}}(n), \ldots, \overline{\tilde{\boldsymbol{v}}}_{Q-1}^{\mathrm{T}}(n)\right]^{\mathrm{T}}$, and relate them into

$$
\overline{\boldsymbol{r}}(n)=e^{\frac{j 2 \pi \nu(k)(n J+P)}{M}} \overline{\boldsymbol{\xi}}^{(k)}+\overline{\boldsymbol{v}}(n) .
$$

Given $\nu^{(k)}$ and $\overline{\boldsymbol{\xi}}^{(k)}$, we can see the vector $\overline{\boldsymbol{r}}(n)$, for $n=0,1 \ldots, N-1$, is Gaussian and independent, with the mean $e^{\left(j 2 \pi \nu^{(k)}(n J+P)\right) /(M)} \bar{\xi}^{(k)}$ and covariance matrix $\sigma_{\tilde{v}}^{2} I_{\left(\bar{M}-\mathcal{K}_{r}\right) Q}\left(\sigma_{\tilde{v}}^{2}\right.$ denotes the noise variance and $\boldsymbol{I}_{\left(\bar{M}-\mathcal{K}_{r}\right) Q}$ 
the identity matrix with the size $\left.\left(\bar{M}-\mathcal{K}_{r}\right) Q \times\left(\bar{M}-\mathcal{K}_{r}\right) Q\right)$. The maximum-likelihood estimate of $\nu^{(k)}$ is

$$
\hat{\nu}^{(k)}=\arg \min _{\hat{\nu}^{(k)}} \sum_{n=0}^{N-1}\left\|\overline{\boldsymbol{r}}(n)-e^{\frac{j 2 \pi \hat{\nu}(k)(n J+P)}{M}} \overline{\boldsymbol{\xi}}^{(k)}\right\|^{2}
$$

where $\|\cdot\|^{2}$ denotes the Frobenius norm. However, as mentioned in [6], solving (19) needs the joint estimation of $\left(\nu^{(k)}, \mu^{(k)}, h^{(k)}\right)$ and may be impractical due to its high computation-complexity. The suboptimal solution is usually used to estimate $\nu^{(k)}$, which is based on the correlation

$$
\begin{aligned}
c(i) & =\frac{1}{N-i} \sum_{n=i}^{N-1} \overline{\boldsymbol{r}}^{\mathcal{H}}(n-i) \overline{\boldsymbol{r}}(n), \quad 1 \leqslant i \leqslant \bar{N} \\
& =e^{\frac{j 2 \pi \hat{\nu}(k)_{J i}}{M}}\left\|\overline{\boldsymbol{\xi}}^{(k)}\right\|^{2}+\eta(i)
\end{aligned}
$$

where $\eta(i)$ is the residual noise, and $\bar{N} \leqslant N-1$. Based on (20), the best linear unbiased estimation of $\nu^{(k)}$ is given by [1], [6]

$$
\hat{\nu}^{(k)}=\frac{M}{2 \pi J} \sum_{i=1}^{\bar{N}} w(i) \angle\left(c(i) c^{*}(i-1)\right)
$$

where $\angle$ stands for the angle, $c(0)=1$

$$
w(i)=\frac{3((N-i)(N-i+1)-\bar{N}(N-\bar{N}))}{\bar{N}\left(4 \bar{N}^{2}-6 N \bar{N}+3 N^{2}-1\right)} .
$$

Given $\nu^{(k)}, \mu^{(k)}, h^{(k)}$, and $\overline{\boldsymbol{U}}_{q}^{\mathcal{H}}$, the suboptimum estimator achieves the minimum MSE (MMSE) for $\bar{N}=N / 2$ (see [6] and [15])

$$
\operatorname{MMSE}\left(\nu^{(k)}\right)=\frac{3 M^{2} Q\left(\bar{M}-\mathcal{K}_{r}\right) \sigma_{\tilde{\tilde{v}}}^{2}}{2 \pi^{2} N\left(N^{2}-1\right) J^{2}\left\|\overline{\boldsymbol{\xi}}^{(k)}\right\|^{2}} .
$$

This bound has been computed considering that the received signal power is $\left\|\overline{\boldsymbol{\xi}}^{(k)}\right\|^{2}$, and the noise power is $E\|\overline{\boldsymbol{v}}(n)\|^{2}=$ $Q\left(\bar{M}-\mathcal{K}_{r}\right) \sigma_{\tilde{v}}^{2}$ ( $E$ for expectation). The estimation range is limited to $\left|\nu^{(k)}\right|<M / 2 J$. The performance loss of the suboptimum estimator can be observed by comparing (23) with the MCRB given by [16]

$$
\operatorname{MCRB}\left(\nu^{(k)}\right)=\frac{3 M^{3} Q\left(\bar{M}-\mathcal{K}_{r}\right) \sigma_{\tilde{v}}^{2}|d(0)|^{2}}{2 \pi^{2}(N J)^{3}\left\|\boldsymbol{\alpha}^{(k)}\right\|^{2}}
$$

where $\boldsymbol{\alpha}^{(k)}=\left[\alpha^{(k)}(0), \alpha^{(k)}(1), \ldots, \alpha^{(k)}(M-1)\right]^{\mathrm{T}}$.

\section{Training Pattern and CFO Identifiability}

Seen from (23), the CFO estimation performance depends on $\left\|\overline{\boldsymbol{\xi}}^{(k)}\right\|$. The optimal training pattern design is to maximize $\left\|\bar{\xi}^{(k)}\right\|$ to achieve the MMSE. However, the optimality needs the knowledge of $\left(\mu^{(k)}, h^{(k)}\right)$ at the transmitter, that is impractical at the CFO estimation procedure. Here, we propose a simple training-pattern design, which targets on the CFO identifiability.

Consider the vector $\overline{\boldsymbol{\xi}}_{q}^{(k)}$ as the following form:

$$
\overline{\boldsymbol{\xi}}_{q}^{(k)}=\underbrace{\overline{\boldsymbol{U}}_{q}^{\mathcal{H}} \boldsymbol{\alpha}_{q}^{(k)}}_{=\overline{\boldsymbol{\alpha}}_{q}^{(k)}}+\underbrace{\overline{\boldsymbol{U}}_{q}^{\mathcal{H}} \boldsymbol{\beta}_{q}^{(k)}}_{=\overline{\boldsymbol{\beta}}_{q}^{(k)}}
$$

where $\boldsymbol{\xi}_{q}^{(k)}=\boldsymbol{\alpha}_{q}^{(k)}+\boldsymbol{\beta}_{q}^{(k)}$, and $\boldsymbol{\alpha}_{q}^{(k)}$ or $\boldsymbol{\beta}_{q}^{(k)}$ is formed by collecting $\alpha^{(k)}(m)$ or $\beta^{(k)}(m)$ [see (11)] in the $q$ th subset. We then define $\overline{\boldsymbol{\alpha}}^{(k)}=\left[\left(\overline{\boldsymbol{\alpha}}_{0}^{(k)}\right)^{\mathrm{T}},\left(\overline{\boldsymbol{\alpha}}_{1}^{(k)}\right)^{\mathrm{T}}, \ldots,\left(\overline{\boldsymbol{\alpha}}_{Q-1}^{(k)}\right)^{\mathrm{T}}\right]^{\mathrm{T}}, \overline{\boldsymbol{\beta}}^{(k)}=$ $\left[\left(\overline{\boldsymbol{\beta}}_{0}^{(k)}\right)^{\mathrm{T}},\left(\overline{\boldsymbol{\beta}}_{1}^{(k)}\right)^{\mathrm{T}}, \ldots,\left(\overline{\boldsymbol{\beta}}_{Q-1}^{(k)}\right)^{\mathrm{T}}\right]^{\mathrm{T}}$, and express $\overline{\boldsymbol{\xi}}^{(k)}$ as

$$
\overline{\boldsymbol{\xi}}^{(k)}=\overline{\boldsymbol{\alpha}}^{(k)}+\overline{\boldsymbol{\beta}}^{(k)} .
$$

Since $\overline{\boldsymbol{\alpha}}^{(k)}$ dominates the CFO estimation performance particularly for the estimation of small CFO, ${ }^{3}$ our training-pattern design is to assure the condition: C1) $\overline{\boldsymbol{\alpha}}^{(k)} \neq \mathbf{0}$.

One possibility of breaking the condition C1) is $\boldsymbol{\alpha}_{q}^{(k)}=\mathbf{0}$, for $q=0,1, \ldots, Q-1$. This case would happen only when all nonzero pilot symbols were corrupted by the channel zeros (deep fades). Since the communication channel (with the order $L_{h}$ ) has at most $L_{h}$ channel zeros, we can avoid this case by employing at least $\left(L_{h}+1\right)$ nonzero pilot symbols in the training block. However, this is not sufficient to guarantee the condition C1), because the filtering matrix $\overline{\boldsymbol{U}}_{q}^{\mathcal{H}}$ does not have the full column rank, and $\overline{\boldsymbol{U}}_{q}^{\mathcal{H}} \boldsymbol{\alpha}_{q}^{(k)}=\mathbf{0}$ is possible even when $\boldsymbol{\alpha}_{q}^{(k)} \neq \mathbf{0}$. To solve this problem, we propose an adaptive training scheme (ATS) as follows.

1) ATS: The proposed training scheme is based on two assumptions: A1) each subset within one training block has $L_{p}$ $\left(\leqslant \bar{M}-K_{r}\right)$ nonzero pilot symbols and A2) $L_{p} \geqslant\left(L_{h}+1\right) / Q$ to combat the channel zeros. Then, we should have

$$
\overline{\boldsymbol{U}}_{q}^{\mathcal{H}} \boldsymbol{\alpha}_{q}^{(k)}=\breve{\bar{U}}_{q}^{\mathcal{H}} \breve{\boldsymbol{\alpha}}_{q}^{(k)}
$$

where $\breve{\boldsymbol{\alpha}}_{q}^{(k)}$ collects $L_{p}$ components in $\boldsymbol{\alpha}_{q}^{(k)}$ corresponding to nonzero pilot symbols, and $\breve{\breve{U}}_{q}^{\mathcal{H}}$ collects the corresponding $L_{p}$ column vectors in $\overline{\boldsymbol{U}}_{q}^{\mathcal{H}}$. Therefore, $\breve{\overline{\boldsymbol{U}}}_{q}^{\mathcal{H}}$ can be adaptively changed according to the placement of nonzero pilot symbols. Since $\overline{\boldsymbol{U}}_{q}^{\mathcal{H}}$ has the full row rank, i.e., $\operatorname{Rank}\left(\overline{\boldsymbol{U}}_{q}^{\mathcal{H}}\right)=$ $\bar{M}-\mathcal{K}_{r}$, we can always find an $\breve{\breve{U}}_{q}^{\mathcal{H}}$ with the full column rank. In this case, the condition C1) does hold for $\left[\left(\breve{\boldsymbol{\alpha}}_{0}^{(k)}\right)^{\mathrm{T}},\left(\breve{\boldsymbol{\alpha}}_{1}^{(k)}\right)^{\mathrm{T}}, \ldots,\left(\breve{\boldsymbol{\alpha}}_{Q-1}^{(k)}\right)^{\mathrm{T}}\right]^{\mathrm{T}} \neq \mathbf{0}$, which is protected by A2). Hence, we can use adaptive placement of nonzero pilot symbols to guarantee the CFO identifiability. The pilot placement information is the feedback from the uplink receiver.

We then consider the extreme case that $\bar{M}-\mathcal{K}_{r}=1$. The assumptions A1) and A2) lead to

$$
L_{p}=1, Q \geqslant L_{h}+1 \text {. }
$$

Since the system setup should be independent of the user-loading condition, we have to use (28) to guarantee the CFO identifiability for arbitrary case. Using the setup (28), $\breve{\boldsymbol{\alpha}}_{q}^{(k)}$ becomes a scalar denoted by $\breve{\alpha}_{q}^{(k)}$, and $\breve{\boldsymbol{U}}_{q}^{\mathcal{H}}$ becomes an $\left(M-\mathcal{K}_{r}\right) \times 1$ vector denoted by $\breve{\boldsymbol{u}}_{q}$. Then, the pilot placement should guarantee $\breve{\boldsymbol{u}}_{q} \neq \mathbf{0}$. We are quite interested in the

\footnotetext{
${ }^{3}$ In the small CFO case, the ICI term $\overline{\boldsymbol{\beta}}^{(k)}$ does not contribute considerably to the CFO estimation. Therefore, the robust training-pattern design should not rely on the contribution from the ICI.
} 
small CFO scenario that the ICI does not affect the estimation performance considerably, i.e.,

$$
\left\|\overline{\boldsymbol{\xi}}^{(k)}\right\|^{2}=\left\|\overline{\boldsymbol{\alpha}}^{(k)}\right\|^{2}=\sum_{q=0}^{Q-1}\left\|\breve{\boldsymbol{u}}_{q}\right\|^{2}\left|\breve{\alpha}_{q}^{(k)}\right|^{2} .
$$

Equation (29) achieves the maximum when $\breve{\boldsymbol{u}}_{q}$ is selected to have the maximum norm among all column vectors in $\bar{U}_{q}^{\mathcal{H}}$. This is the criterion to determine the placement of the nonzero pilot symbol in each subset.

Based on the previous discussion, we conclude that the proposed estimator can offer the robust CFO estimation by employing the ATS under the condition (28). On the other hand, the ATS needs frequent feedback from the uplink receiver if the RU channel as well as the user-loading condition is rapidly changing.

2) Nonadaptive Training Scheme: This training scheme is proposed only for the subband MC-CDMA. Consider an example that the channel fades in the $q$ th subset (subband) are almost identical (see [17]), i.e., $\mathbf{D}\left(\tilde{\boldsymbol{h}}_{q}^{(k)}\right) \approx \tilde{h}_{q}^{(k)} \boldsymbol{I}$. This would happen if the channel is not considerably time dispersive (see example in simulations). The effective RU signatures are expressible as

$$
\tilde{\boldsymbol{C}}_{q} \approx C \mathcal{D}_{q}
$$

where $\boldsymbol{C}=\left[\boldsymbol{c}^{(1)}, \boldsymbol{c}^{(2)}, \ldots, \boldsymbol{c}^{\left(\mathcal{K}_{r}\right)}\right]$, and $\mathcal{D}_{q}=$ $\operatorname{diag}\left\{\tilde{h}_{q}^{(1)}, \tilde{h}_{q}^{(2)}, \ldots, \tilde{h}_{q}^{\left(\mathcal{K}_{r}\right)}\right\}$. Since $C$ is part of the Walsh-Hadamard matrix, we can form a filtering matrix $\overline{\boldsymbol{U}}=\left[\boldsymbol{c}^{\left(\mathcal{K}_{r}+1\right)}, \boldsymbol{c}^{\left(\mathcal{K}_{r}+2\right)}, \ldots, \boldsymbol{c}^{(\bar{M})}\right] / \sqrt{\bar{M}}$, which fulfills $\overline{\boldsymbol{U}}^{\mathcal{H}} \tilde{\boldsymbol{C}}_{q} \approx \mathbf{0}$ and is independent of the subset $\operatorname{index}_{q}$. We can see that column vectors in $\overline{\boldsymbol{U}}^{\mathcal{H}}$ have the identical norm $\sqrt{\left(\bar{M}-\mathcal{K}_{r}\right) / \bar{M}}$. Then, $\left\|\overline{\boldsymbol{\alpha}}^{(k)}\right\|^{2}$ is given by [follow (29)]

$$
\left\|\overline{\boldsymbol{\alpha}}^{(k)}\right\|^{2}=\frac{\bar{M}-\mathcal{K}_{r}}{\bar{M}} \sum_{q=0}^{Q-1}\left|\breve{\alpha}_{q}^{(k)}\right|^{2}>0 .
$$

If the $Q$ nonzero pilot symbols have the identical power $\sigma_{t}$, and are placed over $M$ subcarriers with the equal spacing, then (31) can be further expressed as

$$
\left\|\overline{\boldsymbol{\alpha}}^{(k)}\right\|^{2}=\frac{Q\left(\bar{M}-\mathcal{K}_{r}\right) \sigma_{t}^{2}|d(0)|^{2}}{\bar{M}} \sum_{l=0}^{L_{h}}\left|h^{(k)}(l)\right|^{2} .
$$

Unfortunately, (30) might not hold when the channel becomes more time dispersive. However, the non-ATS may be still valid if $Q$ is sufficiently large that the probability of $\left\|\overline{\boldsymbol{a}}^{(k)}\right\|^{2}=\mathbf{0}$ is very small.

\section{CFO ESTIMATION With INTER-NU-INTERFERENCE}

The system we considered so far, as depicted in Fig. 2(a), does not allow NUs to send the training blocks simultaneously. This restriction effectively avoids the inter-NU-interference, and turns the multiuser scenario into the single-user equivalent scenario while the RUs are filtered out. In this section, we

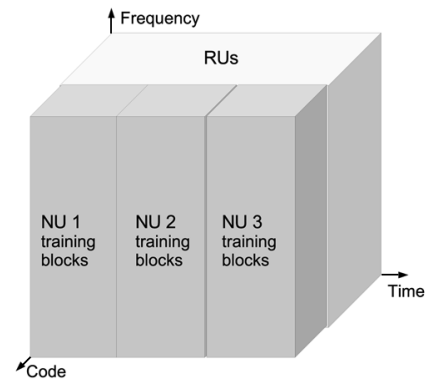

(a)

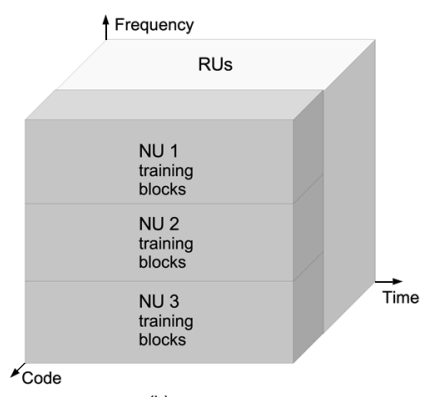

(b)

Fig. 2. Training pattern for MC-CDMA uplink. (a) Example for inter-NU-interference free case. (b) Example with inter-NU-interference.

consider the system that several NUs are allowed to send training blocks simultaneously in the FDMA fashion [see an example in Fig. 2(b)]. The frequency band is divided into $K$ $\mathrm{NU}$ areas denoted by $\mathcal{A}_{k}, k=1,2, \ldots, K$. Each NU area only accommodates one NU.

\section{A. System Model}

We consider the system accommodating $\mathcal{K}_{r}$ RUs as well as $K$ NUs $\left(\mathcal{K}_{r}+K \leqslant \mathcal{K}\right)$. The received time domain signal is expressible as

$$
\begin{aligned}
r(n J+i)= & \underbrace{\left(\sum_{\kappa=1}^{\mathcal{K}_{r}} \bar{r}^{(\kappa)}(n J+i)\right)}_{\mathrm{RUs}} \\
& +\underbrace{\left(\sum_{k=1}^{K} y^{(k)}\left(n J+i-\mu^{(k)}\right)\right)}_{\mathrm{NUs}}+v(n J+i) .
\end{aligned}
$$

As in Section III, we feed $r(n J+i)$ into the OFDM demodulator and obtain

$$
\begin{aligned}
\tilde{r}(n M+m)= & \underbrace{\left(\sum_{\kappa=1}^{\mathcal{K}_{r}} \tilde{h}^{(\kappa)}(m) s^{(\kappa)}(n M+m)\right)}_{=\gamma(n M+m)} \\
& +\left(\sum_{k=1}^{K} e^{\frac{j 2 \pi \nu}{(k)}(n J+P)} \xi^{(k)}(m)\right) \\
& +\tilde{v}(n M+m), 0 \leqslant m \leqslant M-1 .
\end{aligned}
$$


We only consider the received signal within the desired NU area (denoted by $\mathcal{A}_{k_{d}}$ )

$$
\begin{aligned}
\tilde{r}(n M+m)= & \gamma(n M+m)+e^{\frac{j 2 \pi \nu^{\left(k_{d}\right)(n J+P)}}{M}} \xi^{\left(k_{d}\right)}(m) \\
& +\omega(n M+m)+\tilde{v}(n M+m), \\
m \in \mathcal{A}_{k_{d}} &
\end{aligned}
$$

where $\omega(n M+m)$ is the inter-NU-interference term

$$
\begin{aligned}
\omega(n M+m)= & \sum_{k \neq k_{d}} e^{\frac{j 2 \pi \nu^{(k)}(n J+P)}{M}} \\
& \times \sum_{\ell \in \mathcal{A}_{k}} e^{\frac{j 2 \pi \ell \ell^{(k)}}{M}} \tilde{h}^{(k)}(\ell) t^{(k)}(\ell) d(\ell-m) .
\end{aligned}
$$

It is observed that the inter-NU-interference may help the CFO estimation when NUs have the identical CFO. On the other hand, we usually need to cope with distinct CFOs that the inter-NU-interference degrades the estimation performance considerably. The blind approach proposed in [5] supports distinct CFOs indeed but only for the interleaved OFDMA. We also notice that very few training-based approaches have been reported so far in this regard. This motivates us to investigate the inter-NU-interference cancellation in the training-based CFO estimation.

\section{B. Interference-Canceling Training Scheme}

The inter-NU-interference cancellation relies on the trainingpattern design. It is based on the assumptions: A3) subcarriers within each NU area are grouped into $L_{c}$ clusters, and each cluster consists of two adjacent subcarriers and A4) channel fades within each cluster are close. The training symbols within the $l$ th cluster of the $k$ th NU obeys the following criterion:

$$
t^{(k)}(2 l)=-t^{(k)}(2 l+1) .
$$

We apply A3) and A4) as well as (37) in (36) and obtain

$$
\begin{aligned}
\omega(n M+m)= & \sum_{k \neq k_{d}} e^{\frac{j 2 \pi \nu(k)(n J+P)}{M}} \\
& \quad \times \sum_{l=0}^{L_{c}-1} t^{(k)}(2 l) \tilde{h}^{(k)}(2 l) e^{\frac{j 4 \pi l l_{\mu}(k)}{M}} \bar{d}(l)
\end{aligned}
$$

where

$$
\bar{d}(l)=d(2 l-m)-d(2 l+1-m) e^{\frac{j 2 \pi \mu(k)}{M}} .
$$

The idea of using (37) for interference cancellation is not new. It is first proposed by Zhao and Haggman (see [10]) for the ICI self-canceling data-transmission in AWGN downlink channel. One of their major results is

$$
\frac{|d(2 l-m)-d(2 l+1-m)|^{2}}{|d(2 l-m)|^{2}} \approx 10^{-2} .
$$

Here, we borrow the idea for the training-pattern design.

To see the inter-NU-interference canceling capability of the training pattern (37), we need to setup a baseline, i.e., ${ }^{4}$ training symbol is pseudo random and statistically independent with zero mean and the constant amplitude $\sigma_{t}$. Then,

\footnotetext{
${ }^{4}$ The pseudo-random training pattern is employed in many practical systems. The receiver knows the training sequence and can easily make the training sequences to be identical by adjusting the phase.
}

the inter-NU-interference power on the $m$ th subcarrier can be computed from (36)

$$
\begin{aligned}
E|\omega(n M+m)|^{2} & =\sigma_{t}^{2} \sum_{k \neq k_{d}} \sum_{\ell \in \mathcal{A}_{k}}\left|\tilde{h}^{(k)}(\ell)\right|^{2}|d(\ell-m)|^{2}(41) \\
& \approx 2 \sigma_{t}^{2} \sum_{k \neq k_{d}} \sum_{l=0}^{L_{c}-1}\left|\tilde{h}^{(k)}(2 l)\right|^{2}|d(2 l-m)|^{2} .
\end{aligned}
$$

Equation (42) is based on the assumption A4) and $|d(2 l-m)| \approx$ $|d(2 l+1-m)|$ (see [10]). Using the training pattern (37), the training symbols within each cluster are fully correlated, but uncorrelated for different clusters. Then, we can compute the inter-NU-interference for (38)

$$
E|\omega(n M+m)|^{2}=\sigma_{t}^{2} \sum_{k \neq k_{d}} \sum_{l=0}^{L_{c}-1}\left|\tilde{h}^{(k)}(2 l)\right|^{2}|\bar{d}(l)|^{2} .
$$

Assume NUs do not suffer timing offset, i.e., $\mu^{(k)}=0$ for $k=$ $1,2, \ldots, K$. We have $|\bar{d}(l)|=|d(2 l-m)-d(2 l+1-m)|$ and can use (40) to obtain the ratio

$$
\frac{(43)}{(42)} \approx 5 \times 10^{-3} \text {. }
$$

The proposed training pattern shows the strong inter-NU-interference canceling capability. In the presence of timing offset, we consider $|\bar{d}(l)|$ as follows:

$$
\begin{aligned}
|\bar{d}(l)|= & \mid d(2 l-m)-d(2 l+1-m) \\
& +d(2 l+1-m)\left(1-e^{\frac{j 2 \pi \mu^{(k)}}{M}}\right) \mid \\
\leqslant & |d(2 l-m)-d(2 l+1-m)| \\
& +|d(2 l+1-m)|\left|1-e^{\frac{j 2 \pi \mu^{(k)}}{M}}\right| \\
\approx & |d(2 l-m)| \underbrace{\left(0.1+\left|1-e^{\frac{j 2 \pi \mu^{(k)}}{M}}\right|\right)}_{=\eta\left(\mu^{(k)}\right)} .
\end{aligned}
$$

It is observed that $\eta\left(\mu^{(k)}\right)$ is an even function and monotonically increasing for $\mu^{(k)}>0$. Define a timing offset $\bar{\mu}$ fulfilling $|\bar{\mu}|=$ $\max \left\{\left|\mu^{(1)}\right|,\left|\mu^{(2)}\right|, \ldots,\left|\mu^{(K)}\right|\right\}$, (43) is upper bounded by

$E|\omega(n M+m)|^{2} \leqslant \eta^{2}(\bar{\mu}) \sigma_{t}^{2} \sum_{k \neq k_{d}} \sum_{l=0}^{L_{c}-1}\left|\tilde{h}^{(k)}(2 l)\right|^{2}|d(2 l-m)|^{2}$.

Then, the ratio (43)/(42) is upper bounded by

$$
\frac{(43)}{(42)} \leqslant \frac{\eta^{2}(\bar{\mu})}{2} .
$$

We study an example $\bar{\mu} / M=1 / 16$ and use (49) to obtain

$$
\frac{(43)}{(42)} \leqslant 0.12 \text {. }
$$

Comparing (50) with (44), we can see the interference canceling capability of the proposed training pattern is sensitive to the timing offset. But, the performance is still acceptable for small timing offset. 


\section{Training-Pattern Design and CFO Estimation}

We study the training-pattern design for the interleaved MC-CDMA and the subband MC-CDMA respectively, because it is difficult to offer an unified design for both systems.

1) Interleaved $M C$-CDMA: We assume $Q$ to be even ${ }^{5}$ and give the following training-pattern design.

D1) Each subset is shared by all NUs.

D2) The $k$ th NU only has one nonzero pilot symbol within the $q$ th subset (denoted by $t_{q}^{(k)}$ ). $t_{q}^{(k)}$ is placed on the position corresponding to the $(k-1)$ th element in $\xi_{q}^{(k)}$.

D3) $t_{1}^{(k)}=-t_{2}^{(k)}, t_{3}^{(k)}=-t_{4}^{(k)}, \ldots, t_{Q-1}^{(k)}=-t_{Q}^{(k)}$

2) Subband $M C$-CDMA: We give the training-pattern design as follows.

D4) Each subset accommodates up to $\bar{K}_{q}=\lceil(K) /(2)\rceil$ NUs ( $\lceil\cdot\rceil$ for integer ceiling).

D5) Each NU occupies $\lfloor(Q) /(2)\rfloor$ subsets, and has $L_{p}=$ 2 nonzero pilot symbols within each subset.

D6) The two nonzero pilot symbols are placed on the position corresponding to the $2(k-1)$ th and $(2 k-1)$ th elements in $\xi_{q}^{(k)}$ and fulfill the relationship (37).

It is easy to see that the previous designs meet the assumption A3) and (37), and thus, has the inter-NU-interference canceling capability.

3) CFO Estimation: Although the training-pattern design for the MC-CDMA systems is different, the CFO estimation algorithms are almost identical. We consider the received signal within the $q$ th subset, which accommodates $\bar{K}_{q}$ NUs $\left(\bar{K}_{q}=K\right.$ for the interleaved MC-CDMA)

$$
\tilde{\boldsymbol{r}}_{q}(n)=\tilde{\boldsymbol{C}}_{q} \boldsymbol{b}_{q}(n)+\underbrace{\sum_{k=1}^{\bar{K}_{q}} e^{\frac{j 2 \pi \nu(k)(n J+P)}{M}} \boldsymbol{\xi}_{q}^{(k)}}_{=\boldsymbol{\Xi}_{q} \boldsymbol{\nu}(n)}+\boldsymbol{\omega}_{q}(n)+\tilde{\boldsymbol{v}}_{q}(n)
$$

where $\boldsymbol{\omega}_{q}(n)$ denotes the inter-NU-interference within the $q$ th subset, $\boldsymbol{\Xi}_{q}=\left[\boldsymbol{\xi}_{q}^{(1)}, \xi_{q}^{(2)}, \ldots, \boldsymbol{\xi}_{q}^{\left(\bar{K}_{q}\right)}\right]$ and $\boldsymbol{\nu}(n)=\left[e^{\left(j 2 \pi \nu^{(1)}(n J+P)\right) /(M)}, e^{\left(j 2 \pi \nu^{(2)}(n J+P)\right) /(M)}, \ldots\right.$, $\left.e^{\left(j 2 \pi \nu^{\left(\bar{K}_{q}\right)}(n J+P)\right) /(M)}\right]^{\mathrm{T}}$. Then, we use $\bar{U}_{q}^{\mathcal{H}}$ to filter out the RUs and obtain

$$
\overline{\boldsymbol{\boldsymbol { r }}}_{q}(n)=\overline{\boldsymbol{U}}_{q}^{\mathcal{H}} \boldsymbol{\Xi}_{q} \boldsymbol{\nu}(n)+\overline{\boldsymbol{U}}_{q}^{\mathcal{H}} \boldsymbol{\omega}_{q}(n)+\overline{\boldsymbol{\boldsymbol { v }}}_{q}(n) .
$$

For the interleaved MC-CDMA, the training-pattern design D2) leads to

$$
\overline{\boldsymbol{U}}_{q}^{\mathcal{H}} \boldsymbol{\Xi}_{q}=\stackrel{\breve{\boldsymbol{U}}}{q}_{\mathcal{H}}^{\breve{\Xi}}
$$

where $\breve{\Xi}_{q}$ is the $K \times K$ diagonal matrix formed by the first $K$ rows of $\boldsymbol{\Xi}_{q}$, and $\breve{\boldsymbol{U}}_{q}^{\mathcal{H}}$ the $\left(\bar{M}-\mathcal{K}_{r}\right) \times K$ matrix formed by the first $K$ columns of $\overline{\boldsymbol{U}}_{q}^{\mathcal{H}}$. Assuming A5) $\breve{\bar{U}}_{q}^{\mathcal{H}}$ has full-column rank, we multiply $\left(\breve{\bar{U}}_{q}^{\mathcal{H}}\right)^{\dagger}$ with (52) and obtain

$$
\begin{aligned}
\left(\breve{\breve{\boldsymbol{U}}}_{q}^{\mathcal{H}}\right)^{\dagger} \overline{\boldsymbol{\boldsymbol { r }}}_{q}(n)=\boldsymbol{\Xi}_{q} \boldsymbol{\nu}(n)+\left(\breve{\overline{\boldsymbol{U}}}_{q}^{\mathcal{H}}\right)^{\dagger} & \overline{\boldsymbol{U}}_{q}^{\mathcal{H}} \boldsymbol{\omega}_{q}(n) \\
& +\left(\breve{\overline{\boldsymbol{U}}}_{q}^{\mathcal{H}}\right)^{\dagger} \overline{\boldsymbol{\boldsymbol { v }}}_{q}(n) .
\end{aligned}
$$

${ }^{5}$ If $Q$ is odd, then we use $Q-1$ subsets.
Then, the CFO estimation for NUs can be carried out individually by employing the $\mathrm{CFO}$ estimator developed for the inter-NU-interference free case. For the subband MC-CDMA, we can use D6) to find the relationship (53). But, here, $\breve{\Xi}_{q}$ is a $2 \bar{K}_{q} \times \bar{K}_{q}$ matrix. Under the assumption A5), (54) is still valid for the subband MC-CDMA.

We can see that the CFO identifiability needs the assumption A5). However, the ATS is not applicable here to protect this assumption. Therefore, we expect $Q$ to be sufficiently large so that we could have at least one valid subset.

\section{Simulations AND Discussions}

Computer simulations used MSE, $E|\hat{\nu}-\nu|^{2}$, to evaluate the proposed CFO estimation approach, and bit-error-rate (BER) to examine the impact on the overall system performance. The results were obtained by averaging over 500 independently generated multiuser channels with the maximum delay of $2.51 \mu \mathrm{s}$, which comply with the 3GPP Vehicular A model specified by [18]. The near-far effect ([19]) was assumed to be well compensated via power control at the user end. The signal-to-noise ratio (SNR) was defined by the average received signal energy to noise. The setup of MC-CDMA system was given by: $M=64$ subcarriers, and each $\mathrm{CP}$ has $P=4$ samples $(J=M+$ $P=68)$. The user signatures were drawn from the $4 \times 4$ Walsh-Hadamard matrix, i.e., $\bar{M}=4$ and $Q=16$. The information-bearing symbols were drawn from the QPSK constellation with the equal probability. The timing offset for each NU were randomly generated according to the uniform distribution with $\left|\mu^{(k)} / M\right|<1 / 16$. Throughout the simulations, each NU sent $N=4$ training blocks for the CFO estimation. We divide the simulations into the following four concrete experiments.

Experiment 1 (Subband MC-CDMA, Inter-NU-Interference Free): The objective of this experiment is to evaluate the proposed CFO estimation approach together with the training-pattern design in the subband MC-CDMA system that only one NU is allowed to enter the network. The OFDM block duration (exclusive CP) is set to be 80 or $40 \mu \mathrm{s}$. The normalized CFO is considered $\nu^{(k)}=-0.01$ (the small-CFO case) or $\nu^{(k)}=0.2$ (the large-CFO case). There are three RUs already in the network. To show the significance of the training-pattern design, we also investigate the estimation performance that the pilot power is uniformly allocated over all subcarriers (called uniform pilot placement (UPP) for convenience).

We first study the estimation performance for the OFDM block duration of $80 \mu$ s that each subset does not experience the considerably frequency-selective fade. The MSE results for $\nu^{(k)}=-0.01$ are illustrated in Fig. 3. It is observed that the UPP training-pattern does not offer the successful CFO estimation. This is because the improperly designed training-pattern, e.g., UPP, cannot guarantee the CFO identifiability and offer the robust $\mathrm{CFO}$ estimation. The same result is obtained in other simulations in the subband MC-CDMA system. When the proposed training patterns (ATS and non-ATS) are employed, Fig. 3 shows that the MSE bounds for both training patterns are almost identical. The simulation results converge to the MSE bounds in the high-SNR range (e.g., $>16 \mathrm{~dB}$ ). The non-ATS shows faster convergence than the ATS, and thus, offers better estimation performance in the medium-SNR range. We also 


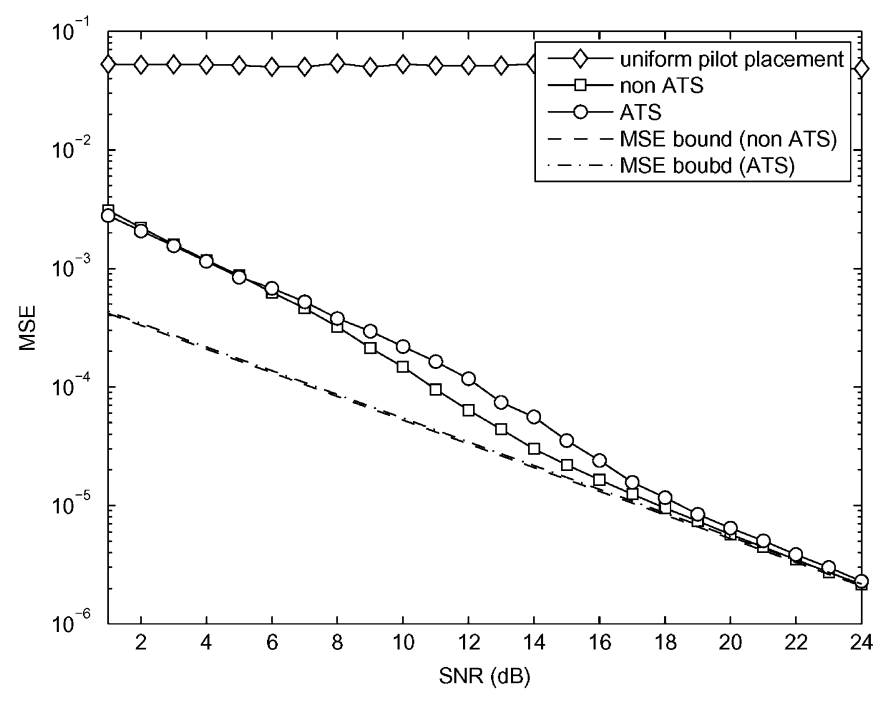

Fig. 3. MSE versus SNR in inter-NU-interference free case, subband MC-CDMA, $\nu^{(k)}=-0.01$.

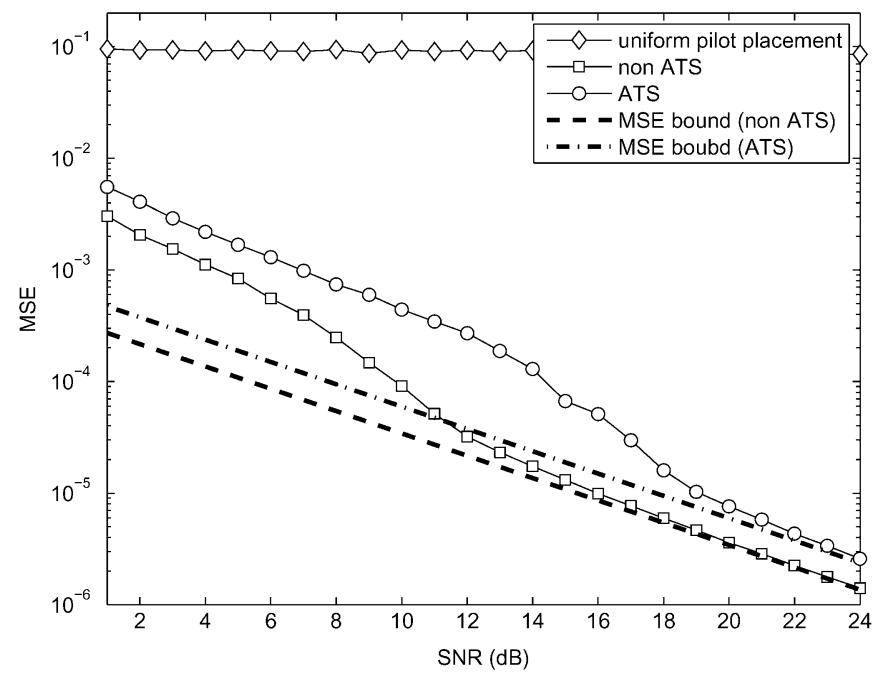

Fig. 4. MSE versus SNR in inter-NU-interference free case, subband MC-CDMA, $\nu^{(k)}=0.2$.

plot the results for $\nu^{(k)}=0.2$ in Fig. 4. It is shown that the non-ATS outperforms the ATS in both the estimation performance and the convergence. It means that the ICI power shows the positive impact on the CFO identifiability.

We then shorten the OFDM block duration to $40 \mu$ s (equivalent to double the subcarrier spacing). The channel within each subset becomes more frequency selective. The MSE results are plotted in Figs. 5 and 6 regarding the different CFO setup. For the case of $\nu^{(k)}=-0.01$ (see Fig. 5), the ATS slightly improves the estimation performance in comparison to the nonATS. However, the non-ATS outperforms the ATS for the case of $\nu^{(k)}=0.2$. It is also observed that both training schemes have the close convergence-rate to the MSE bounds.

As a summary, we have shown that both ATS and non-ATS may offer the robust CFO estimation in the sub-band MC-CDMA. On the other hand, the ATS is preferred here due to its avoiding the feedback as well as the relatively fast convergence to the MSE bound.

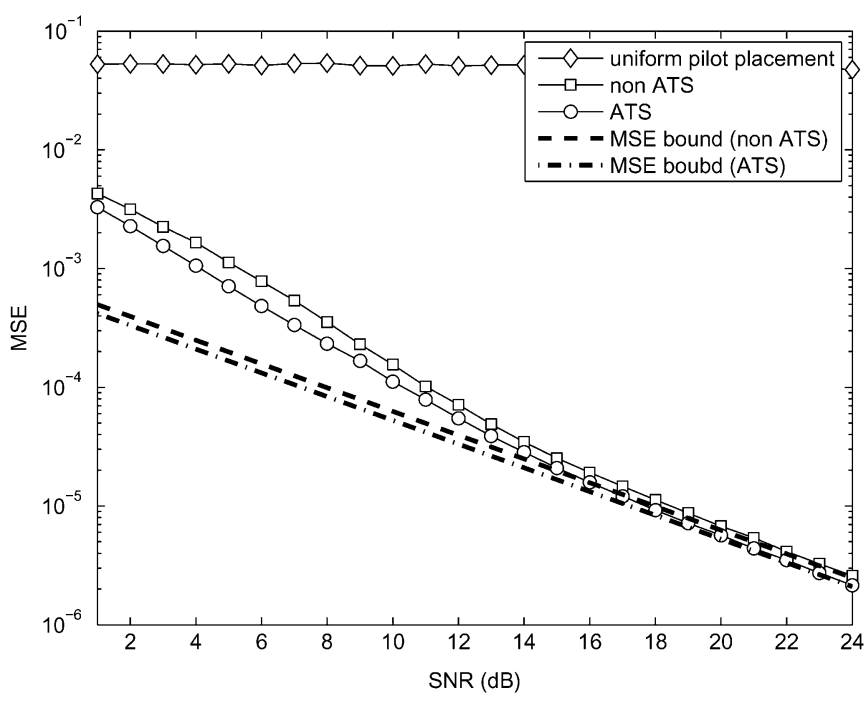

Fig. 5. MSE versus SNR in inter-NU-interference free case, subband MC-CDMA, $\nu^{(k)}=-0.01$.

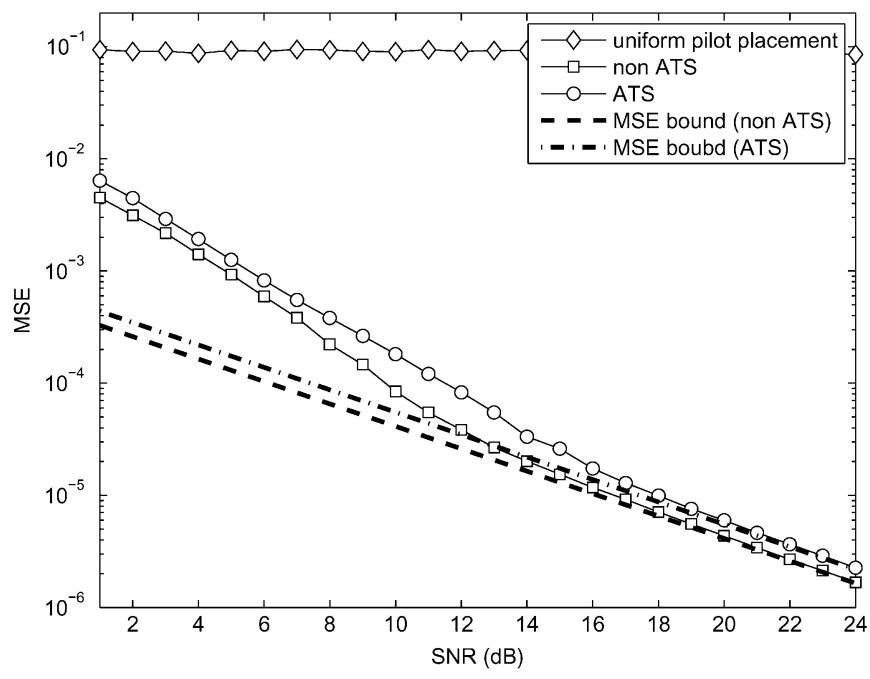

Fig. 6. MSE versus SNR in inter-NU-interference free case, subband MC-CDMA, $\nu^{(k)}=0.2$.

Experiment 2 (Interleaved MC-CDMA, Inter-NU-Interference Free): In this experiment, the RUs use the interleaved MC-CDMA as the air-interface. As mentioned in Sections II and III, each subset experiences the severe frequency-selective fade. Starting from this experiment, the OFDM block duration is fixed to $40 \mu \mathrm{s}$. Other parameters are the same as in Experiment 1 . We first examine the estimation performance for $\nu^{(k)}=-0.01$, and plot the MSE results in Fig. 7. It is interesting to see that the UPP works, but shows the worst performance (around $4 \mathrm{~dB}$ loss to the non-ATS and $12 \mathrm{~dB}$ loss to the ATS). The MSE bounds together with the simulation results show that the ATS can significantly improve the estimation performance in comparison to the non-ATS $(6 \mathrm{~dB}$ improvement in the MSE bound). The ATS converges to the MSE bound for SNR $>16 \mathrm{~dB}$. The non-ATS does not offer the close performance to the MSE bound even for SNR $=24 \mathrm{~dB}$.

We then consider the case of $\nu^{(k)}=0.2$ and plot the results in Fig. 8. It is observed from the MSE bounds that the difference 


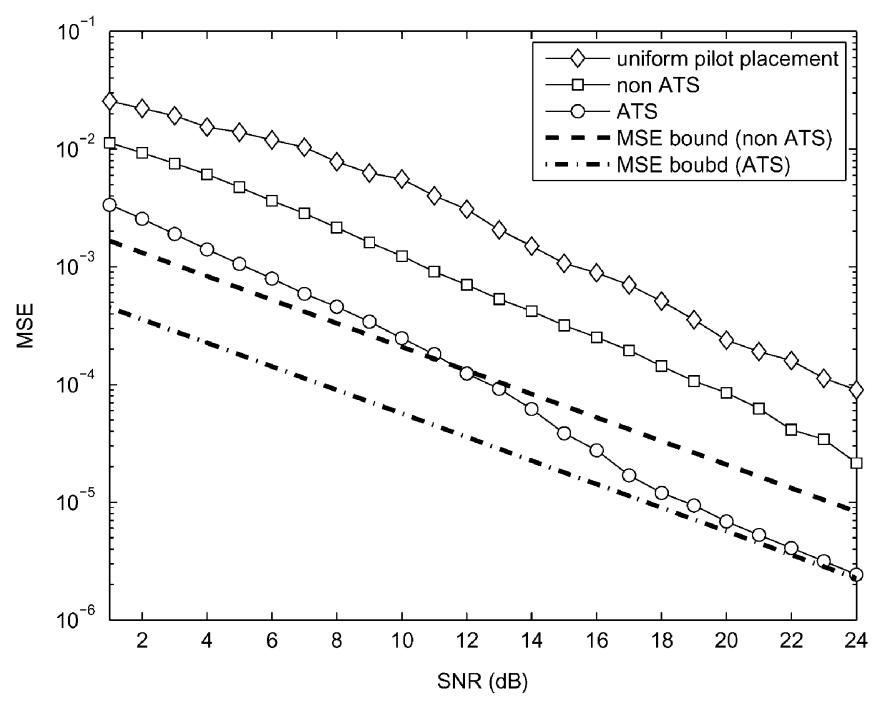

Fig. 7. MSE versus SNR in inter-NU-interference free case, interleaved MC-CDMA, $\nu^{(k)}=-0.01$.

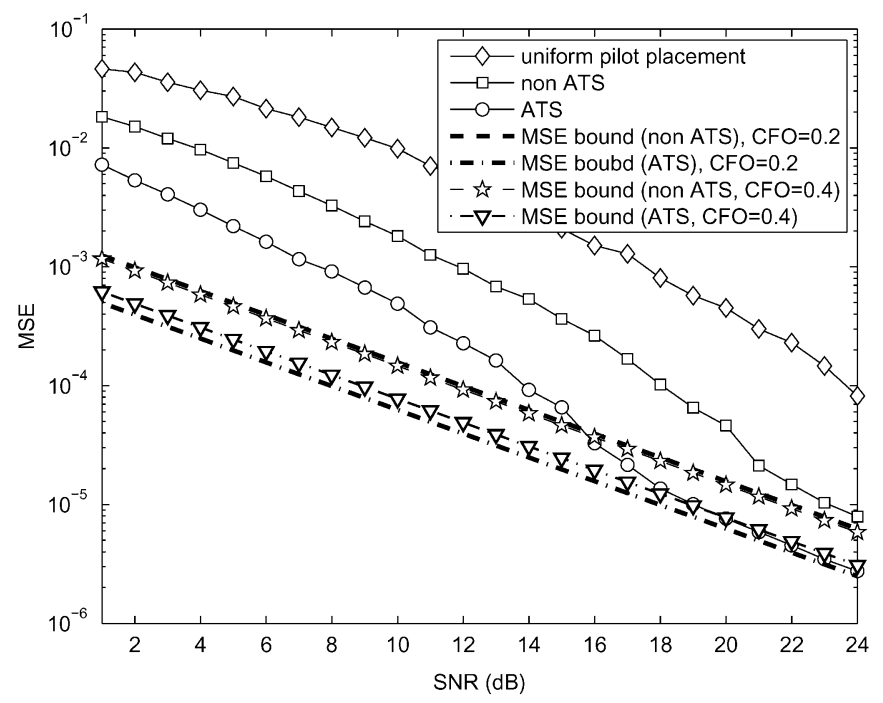

Fig. 8. MSE versus SNR in inter-NU-interference free case, interleaved MC-CDMA, large CFO.

between ATS and non-ATS is reduced to $4 \mathrm{~dB}$. The non-ATS converges to its MSE bound for SNR $>21 \mathrm{~dB}$. In order to observe whether the difference can be further reduced with the increase of CFO, we also plot the MSE bounds for $\nu^{(k)}=0.4$. It can be seen that the reduction of the difference is small $(<$ $1 \mathrm{~dB}$ ).

We summarize that the non-ATS does not offer the robust CFO estimation in the interleaved MC-CDMA particularly for the small-CFO case. The ATS approach is much suitable here for its considerable MSE gain.

Experiment 3 (Final Ber Performance): The objective of this experiment is to examine the final BER performance with the $\mathrm{CFO}$ and timing-offset compensation at the transmitter. It might be interesting to see in the interleaved MC-CDMA whether the MSE gain obtained from the ATS approach considerably improves the final BER performance. We consider there are three active-users in the network with the normalized CFOs of $-0.01,0.2$, and 0.4 , respectively. The uplink receiver has

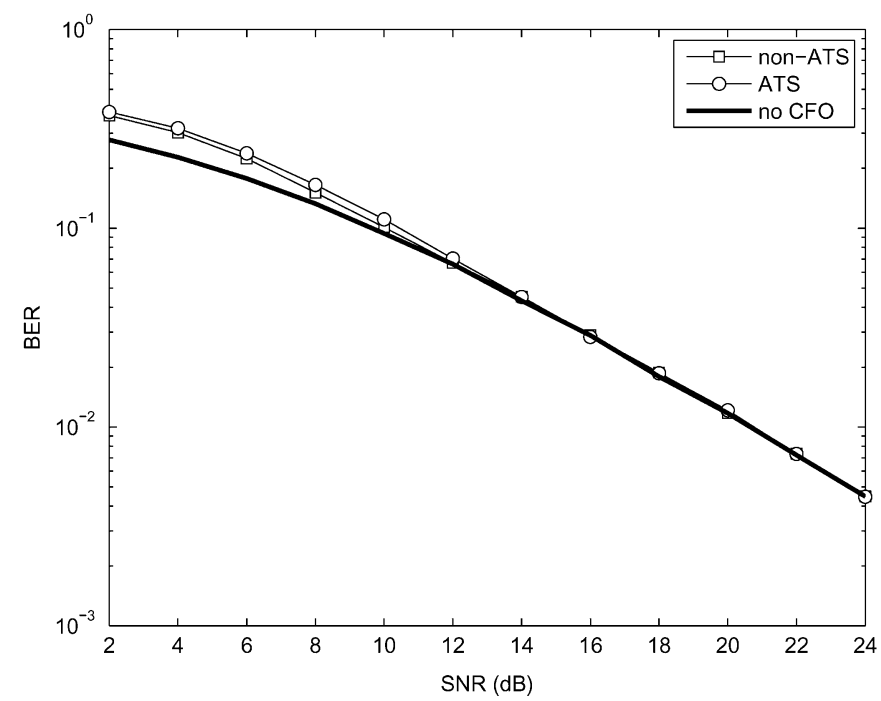

Fig. 9. Final BER versus SNR in inter-NU-interference free case, subband MC-CDMA.

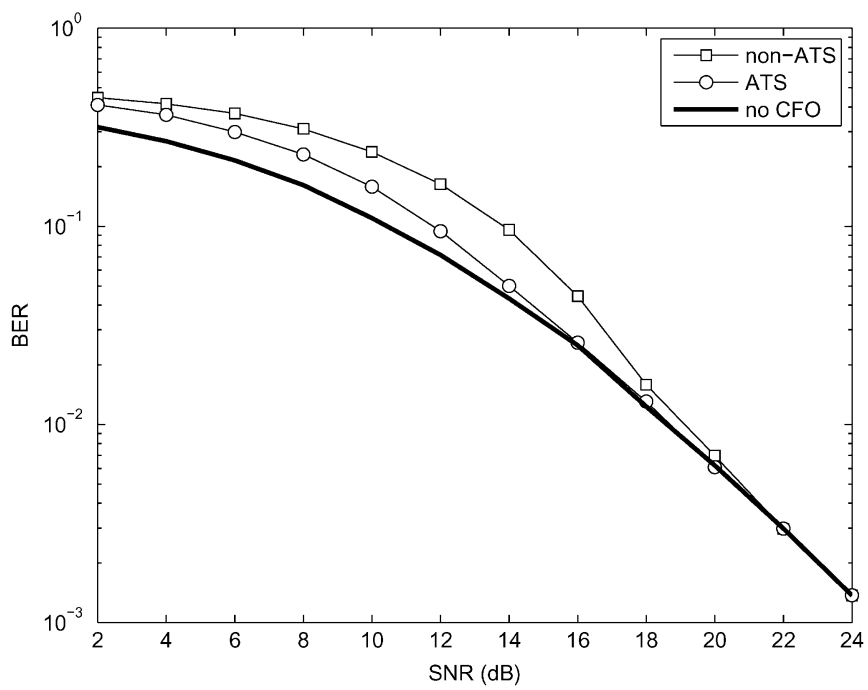

Fig. 10. Final BER versus SNR in inter-NU-interference free case, interleaved MC-CDMA.

the perfect channel knowledge and employs the ZF equalizer (7) for the multi-user channel equalization. Each user has the perfect knowledge of the timing offset. The CFO knowledge at the user-end is the CFO estimate fed back by the uplink receiver. The baseline for the performance comparison is the case of null CFO. It is observed that the BER performance for the three users is almost identical. Therefore, we only plot the simulation results for the user with $\nu=0.2$ as an example.

Fig. 9 illustrates the final BER in the subband MC-CDMA system. We can see that both ATS and non-ATS offer almost identical BER. Their performance converges to the baseline for SNR $>12 \mathrm{~dB}$. The non-ATS shows slightly BER improvement for $\mathrm{SNR}<12 \mathrm{~dB}$. Therefore, in the viewpoint of BER, we also suggest to use the non-ATS approach in the subband MC-CDMA.

Fig. 10 illustrates the final BER in the interleaved MC-CDMA system. It shows that the ATS outperforms the non-ATS. The performance improvement is around $2 \mathrm{~dB}$ 


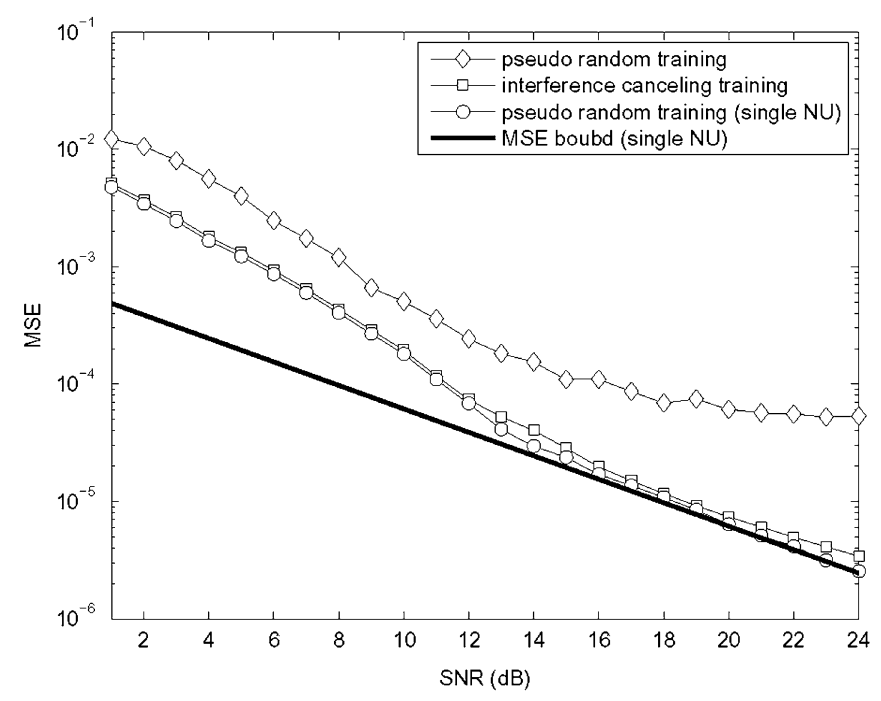

Fig. 11. MSE versus SNR in inter-NU-interference case, subband MC-CDMA.

for the typical SNR $(=12 \mathrm{~dB})$. The ATS offers the almost identical performance to the baseline for SNR $>14 \mathrm{~dB}$. On the other hand, the non-ATS offers the comparable performance to the ATS as well as the baseline for SNR $>18 \mathrm{~dB}$. Therefore, the non-ATS may be the suitable approach in the high-SNR scenario for its avoiding the frequent feedback.

Experiment 4 (Inter-NU-Interference Scenario): The objective of this experiment is to examine the proposed inter-NU-interference canceling training pattern. We consider the simulation example that two RUs already in the network. Two NUs send the training blocks simultaneously for the CFO estimation. We only consider the CFO estimation for the desired NU whose normalized CFO is set to be 0.2 . The other NU is considered the interfering NU whose normalized CFO is randomly generated according to the uniform distribution within the range of $[-0.45,0.45]$. We also consider two baselines for the performance comparison. The first baseline is based on the pseudo-random training pattern. The second baseline also uses the pseudo-random training pattern, but the CFO for the interfering NU is zero. Therefore, the second baseline is actually the single-NU case.

Fig. 11 illustrates the MSE results in the subband MC-CDMA system. We can see that the pseudo-random training pattern shows the worst performance. An error-floor is observed for SNR $>12 \mathrm{~dB}$. The performance loss in comparison to the MSE bound is up to $14 \mathrm{~dB}$. It means that the inter-NU-interference severely affects the CFO estimation. We can also see that the proposed training pattern offers very close performance to the single-NU case. The performance loss in comparison to the MSE bound is up to $1 \mathrm{~dB}$ (in the high-SNR range). The considerable MSE gain is due to the inter-NU-interference canceling capability of the proposed training pattern.

Fig. 12 illustrates the MSE results in the interleaved MC-CDMA system. Again, the pseudo-random training pattern shows the worst performance (or does not offer the successful $\mathrm{CFO}$ estimation). The proposed training pattern offers the comparable performance to the single-NU case for SNR $<14 \mathrm{~dB}$. However, an error floor is observed in the high-SNR range.

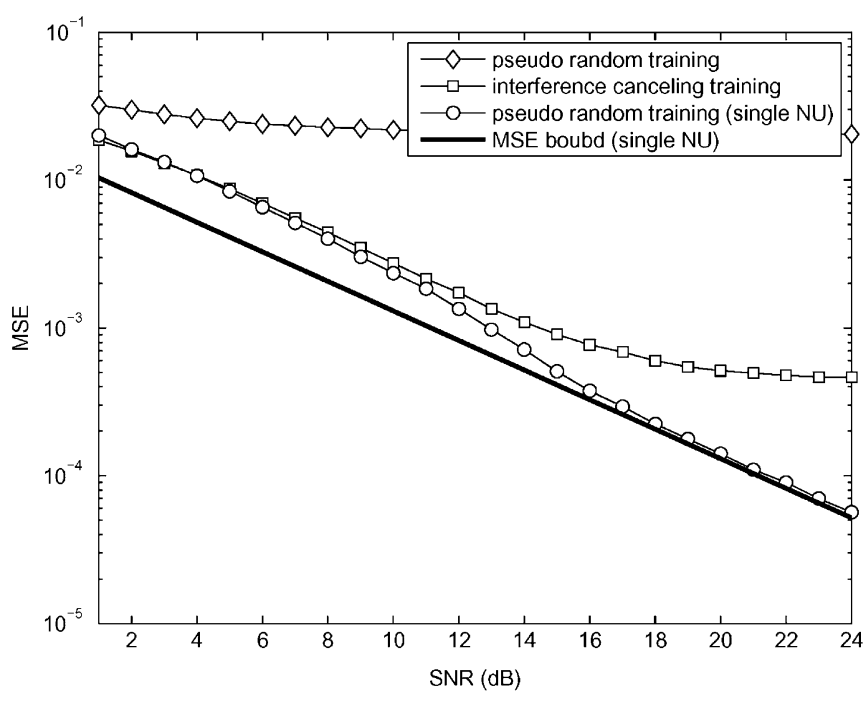

Fig. 12. MSE versus SNR in inter-NU-interference case, interleaved MC-CDMA.

\section{CONCLUSION}

In this paper, we have presented a novel training-based CFO estimation method for the MC-CDMA uplink. The proposed approach exploited the SVD to find the desired subspace that was orthogonal to the RU subspace. The optimum or suboptimum CFO estimation algorithm could be applied in the desired subspace. We have investigated the proposed approach both in the subband and the interleaved MC-CDMA systems, and shown that the CFO identifiability need the proper training-pattern (or training scheme) design. Then, both ATS and non-ATS training schemes were proposed and carefully investigated via both theoretical and simulation analysis. It has been shown that the non-ATS is suitable for the subband MC-CDMA system or the interleaved MC-CDMA system in the high-SNR scenario. The ATS is suitable for the interleaved MC-CDMA in the small and medium-SNR range.

We also investigated the proposed CFO estimation approach in the inter-NU-interference scenario. It has been shown that the inter-NU-interference severely affected the CFO estimation. Therefore, we proposed the inter-NU-interference self-canceling training pattern and investigated its interference canceling capability in the Gaussian channel. Simulation results have shown that, in the subband MC-CDMA, the proposed training pattern could offer the comparable performance to the single-NU case. In the interleaved MC-CDMA, it demonstrated the comparable performance to the single-NU case in the small and medium-SNR range.

\section{ACKNOWLEDGMENT}

The authors would like to thank the Associate Editor, Prof. E. Serpedin, and the reviewers for the efficient review process and constructive comments.

\section{REFERENCES}

[1] U. Mengali and M. Morelli, "Data-aided frequency estimation for burst digital transmission," IEEE Trans. Commun., vol. 45, no. 1, pp. 23-25, Jan. 1997. 
[2] X. Ma, C. Tepedelenlioglu, and G. B. Giannakis, "Non-data-aided carrier offset estimator for OFDM with null subcarriers: Identifiability, algorithms, and performance," IEEE J. Select. Areas Commun., vol. 19, no. 12, pp. 2504-2515, Dec. 2001.

[3] J. J. van de Beek, "A time and frequency synchronization scheme for multiuser OFDM," IEEE J. Select. Areas Commun., vol. 17, no. 11, pp. 1900-1914, Nov. 1999.

[4] J. J. van de Beek, M. Sandell, and P. O. Borjesson, "ML estimation of timing and frequency offset in OFDM systems," IEEE Trans. Signal Process., vol. 45, no. 7, pp. 1800-1805, Jul. 1997.

[5] Z. Cao, U. Tureli, and Y. Yao, "Deterministic multiuser carrier frequency offset estimation for interleaved OFDMA uplink," IEEE Trans. Commun., vol. 52, no. 9, pp. 1585-1594, Sep. 2004.

[6] M. Morelli, "Timing and frequency synchronization for uplink of an OFDMA system," IEEE Trans. Commun., vol. 52, no. 2, pp. 296-306, Feb. 2004

[7] Z. Wang and G. B. Giannakis, "Wireless multicarrier communications: Where Fourier meets Shannon," IEEE Signal Process. Mag., vol. 17, no. 3, pp. 29-48, May 2000.

[8] S. Kaiser and K. Fazael, "A flexible spread-spectrum multi-carrier multiple-access system for multi-media applications," in Proc. IEEE PIMRC, 1997, pp. 100-104.

[9] S. Hara and R. Prasad, "Overview of multicarrier CDMA," IEEE Commun. Mag., vol. 35, no. 12, pp. 126-133, Dec. 1997.

[10] Y. Zhao and S. G. Haggman, "Intercarrier interference self-cancellation scheme for OFDM mobile communication systems," IEEE Trans. Commun., vol. 49, no. 7, pp. 1185-1191, Jul. 2001.

[11] J. Proakis, Digital Communications, 4th ed. New York: McGrawHill, 2001.

[12] X. Cai, S. Zhou, and G. B. Giannakis, "Group-orthogonal multicarrier CDMA,” IEEE Trans. Commun., vol. 52, no. 1, pp. 90-99, Jan. 2004.

[13] T. Pollet, M. V. Bladel, and M. Moeneclaey, "BER sensitivity of OFDM systems to carrier frequency offset and Wiener phase noise," IEEE Trans. Commun., vol. 43, no. 2, pp. 191-193, Feb. 1995.

[14] G. H. Golub and C. F. Van Loan, Matrix Computations, 3rd ed. London, U.K.: Johns Hopkins Univ. Press, 1996

[15] M. Morelli, "An improved frequency offset estimator for OFDM application," IEEE Commun. Lett., vol. 3, no. 3, pp. 75-77, Mar. 1999.

[16] A. N. D'Andrea, U. Mengali, and R. Reggiannini, "The modified Cramér-Rao bound and its application to synchronization problems," IEEE Trans. Commun., vol. 42, no. 2, pp. 1391-1399, Feb./Apr. 1994.
[17] X. Gui and T. S. Ng, "Performance of asynchronous orthogonal multicarrier CDMA system in frequency selective fading channel," IEEE Trans. Commun., vol. 47, no. 7, pp. 1084-1091, Jul. 1999.

[18] 3rd Generation Partnership Project; Technical Specification Group Radio Access Network; Spatial Channel Model for Multiple Input Multiple Output (MIMO) Simulations (Release 6), 3GPP TR 25.996 v6.1.0, Sep. 2003.

[19] T. S. Rappaport, Wireless Communications: Principles and Practice. Englewood Cliffs, NJ: Prentice-Hall, 1996.

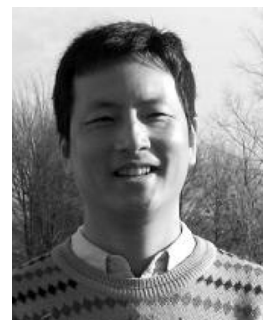

Yi Ma (M'04) is currently a Postdoctoral Research Fellow at the Centre for Communication Systems Research (CCSR), the University of Surrey, Surrey, U.K. His research interests include statistical signal processing, modulation, estimation, detection and synchronization theory in wireless communications, network information theory, and random matrix theory.

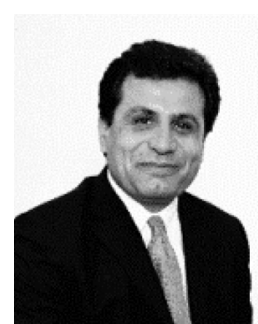

Rahim Tafazolli is a Professor of Mobile/Personal communication and Head of Mobile Communications Research Group and is also the Director of Research in the School of EEITM, the University of Surrey, Surrey, U.K. He has been active in research for 20 years and has authored and co-authored more than 300 papers in referred international journals and conferences. He has been consultant to many mobile companies, lectured and chaired a number of IEE Summer schools and IEEE workshops and conferences. He has carried out many consultancies for mobile companies, Home Office, and European Union all in the field of mobile communications. He is the Founder and Chairman of IEE International Conference on Third Generation Mobile Communications. 